\title{
Contribution à la connaissance des micromycètes du Maroc. Cas des espèces en survie dans un milieu aquatique
}

\author{
Aouatif BOUSRHAL, Hafida El OMARI, Mohamed RAHOUTI", \\ Aïcha El AISSAMI et Amina REgRAGUI
}

Faculté des sciences, Département de biologie, Avenue Ibn Battouta, BP 1014, 10000 Rabat, Maroc.

*Correspondance,courriel:rahouti@fsr.ac.ma

\section{Résumé}

Cette étude présente les premiers résultats concernant l'analyse quantitative et qualitative des micromycètes d'origine terrestre de la région de Rabat (Maroc). Les espèces fongiques décrites ont été isolées de l'eau d'un lac-réservoir; elles ont été entrainées dans le milieu aquatique par différents moyens de transport.

L'analyse fongique des échantillons d'eau prélevés du rivage et du large du lac, ainsi que de l'écume et d'autres matériaux immergés dans l'eau a montré que l'eau du lac est riche en champignons (10 $250 \mathrm{UFC} /$ litre). Sa charge fongique n'est pas uniformément répartie ; c'est l'eau du rivage qui est la plus chargée.

Les souches isolées sont identifiées en se basant sur la morphologie de leurs appareils végétatif et reproducteur. Cinquante espèces de micromycètes ont été obtenues; elles appartiennent principalement aux genres Penicillium, Fusarium, Aspergillus et Trichoderma. Ce sont surtout des champignons telluriques, en survie dans l'eau et à large répartition géographique. Six de ces espèces sont inventoriées pour la première fois au Maroc.

Mots-clés : Fusarium, Penicillium, micromycètes, champignons terrestres, lac-réservoir, Maroc. 


\section{Abstract \\ Contribution to knowledge of the micromycetes of Morocco. Species survival in an aquatic environment}

The principal aim of this study was to present first results concerning the quantitative and qualitative analysis of micromycetes of terrestrial origin from the region of Rabat (Morocco). The described fungal species have been isolated from water of a lakereservoir; they were driven in the aquatic environment by different means of transportation.

The fungal analysis of water samples collected from beach and large of the lake, as well as of the foam and other materials immersed in water showed that the lake water is rich in fungi (10 $250 \mathrm{UFC} /$ liters). Its fungal load is not distributed uniformly; the beach water is the most loaded.

The isolated strains were identified on the basis on morphology of their vegetative and reproductive structures. Fifty species of micromycetes have been identified; they mainly belong to the genera of Penicillium, Fusarium, Aspergillus and Trichoderma. These are especially terrestrial fungi in survival in water and have a large geographical distribution. Six species are inventoried for the first time in Morocco.

Keywords : Fusarium, Penicillium, micromycetes, terrestrial fungi, lake-reservoir,

\section{Morocco.}

\section{Introduction}

L'étude systématique de la mycoflore du Maroc n'a été abordée que d'une façon sporadique; c'est pour cela qu'elle reste encore mal connue. En ce qui concerne les Basidiomycètes, la contribution la plus importante est celle de Malençon [1-4] et Guyot \& Malençon [5,6]. Dans les tomes I et II de la flore des champignons supérieurs du Maroc, Malençon \& Bertault $[7,8]$ ont étudié en détail les Agaricales. Les autres groupes de champignons et en particulier ceux qui portent des fructifications microscopiques ou micromycètes n'ont reçu que peu d'attention. Parmi ces derniers, ce sont principalement quelques espèces pathogènes de plantes cultivées qui ont été traitées [9-11] et une centaine d'espèces recensées par Sage et al. [12].

Les espèces de micromycètes décrites dans ce travail sont issues de milieux terrestres et ont été entrânées par différents moyens dans l'eau douce du lac-réservoir Sidi 
Mohammed Ben Abdellah (SMBA) ; elles ont été isolées de ce milieu au cours d'une étude multidisciplinaire visant à rechercher les facteurs biotiques susceptibles d'altérer la qualité de l'eau de ce lac. L'eau de la retenue SMBA est destinée à la production d'eau potable.

Le but du présent travail est donc de contribuer à la connaissance des micromycètes du Maroc en présentant les premiers résultats concernant leur diversité taxonomique et leur abondance dans un milieu aquatique.

\section{Matériel et méthodes}

\section{2-1. Localisation des sites de prélèvement}

Le lac-réservoir SMBA, situé à une vingtaine de kilomètres de la ville de Rabat (Maroc), est alimenté par trois oueds ( Bouregreg, Grou et Korifla ; Carte 1 ). II a été mis en eau en 1974 ; la surface de son plan d'eau est de $32 \mathrm{~km}^{2}$. Sa végétation aquatique ou terrestre environnante est réduite.

Quatre sites ont été choisis dans le lac-réservoir pour l'échantillonnage de l'eau. Les deux premiers sont situés au niveau du rivage, l'un à la surface de l'eau et l'autre au fond à $0,5 \mathrm{~m}$ de profondeur. Les deux autres sites sont localisés au dessous du point représentatif ; l'un au niveau de la thermocline à environ $9 \mathrm{~m}$ de profondeur et l'autre à un mètre du fond. Ce point, considéré comme représentatif de la masse d'eau de la retenue, a été repéré suite à de nombreuses campagnes d'échantillonnage et d'analyses chimiques et microbiologiques par la Direction de Contrôle de la Qualité des Eaux de l'Office National de l'Eau Potable du Maroc; il est localisé près de la tour de prise à environ $500 \mathrm{~m}$ du corps du barrage. 


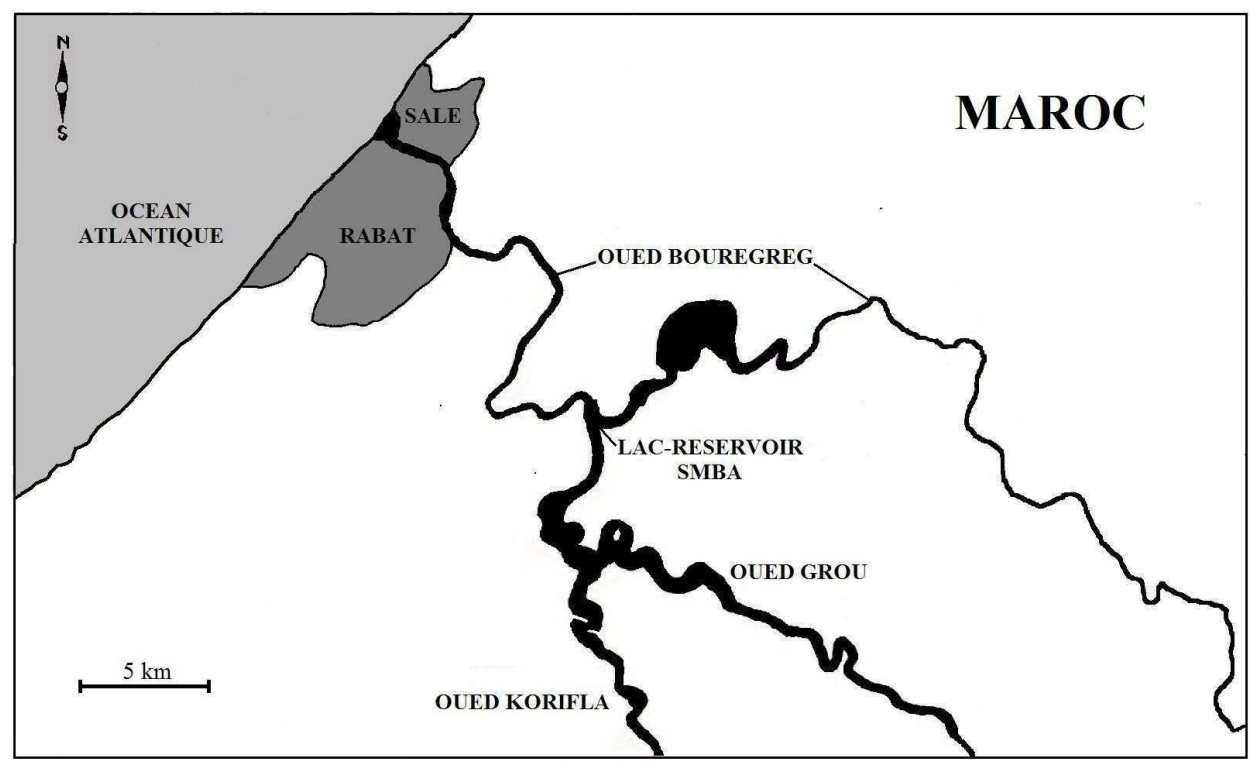

Carte 1 : Localisation géographique des oveds alimentant le lac-réservoir SMBA.

\section{2-2. Période et techniques d'échantillonnage}

Quinze campagnes de prélèvements ont été menées au lac-réservoir SMBA; elles ont débuté en juin 2001 et ont pris fin en novembre 2004.

Les méthodes de prélèvement mises en œuvre diffèrent selon la profondeur de l'eau à analyser. L'eau de surface est recueillie directement dans des flacons. L'échantillonnage en profondeur est réalisé à l'aide de la bouteille de Ruttner. En parallèle avec les échantillons d'eau, d'autres matériaux présents dans le milieu aquatique sont prélevés ; ce sont des écumes, des débris végétaux et un sédiment de nature organique provenant essentiellement de la décomposition des algues mortes. Le matériel utilisé pour l'échantillonnage est propre et stérile. Les prélèvements sont acheminés au laboratoire à basse température et au cours de la même journée.

\section{2-3. Culture et isolement des micromycètes}

Les techniques et les milieux de culture mis en œuvre pour isoler les champignons sont classiques. Les milieux de culture retenus sont gélosés à $1,5 \%$; il s'agit du milieu à base d'extrait de malt à $1,5 \%$ et du milieu P.D.A. (Potato Dextrose Agar). La prolifération des bactéries dans les cultures est inhibée par l'addition de chloramphénicol aux milieux à 
raison de $0,5 \mathrm{~g} / \mathrm{L}$ [12]. Le matériel et les milieux utilisés pour la culture sont stérilisés par autoclavage à $120^{\circ} \mathrm{C}$ pendant 20 minutes.

Les échantillons d'eau et d'écume sont étalés à la surface des milieux gélosés; les débris végétaux et le sédiment organique sont incorporés aux milieux de culture. Les cultures ensemencées sont ensuite incubées à $25^{\circ} \mathrm{C}$ dans l'obscurité et sont examinées chaque jour pour apprécier la croissance des microorganismes.

\section{2-4. Dénombrement des champignons}

L'échantillon liquide à dénombrer est d'abord homogénéisé par agitation; ensuite un volume déterminé en est prélevé pour être, si besoin est, dilué dans l'eau distillée stérile. Un $\mathrm{mL}$ de la solution obtenue est incorporé dans $15 \mathrm{~mL}$ de gélose à l'extrait de levure au dextrose et au chloramphénicol [13]. Au moment de l'incubation, les propagules présentes dans l'échantillon forment des colonies visibles. Ces dernières sont comptées et le résultat final est exprimé en nombre d'unité formant colonie (UFC) par unité de volume de l'échantillon.

\section{2-5. Purification et identification des souches}

Les souches isolées sont soumises à la purification pour les débarrasser de leurs contaminants. La purification est réalisée par repiquages successifs sur milieux gélosés d'une petite bouture du thalle ou de quelques spores. Les espèces fongiques sont identifiées à l'aide d'ouvrages de la systématique fongique [14-21] en se basant sur leurs caractères culturaux macroscopiques et leur morphologie observée sous microscope.

\section{Résultats et discussion}

\section{3-1. Dénombrement des champignons}

Le nombre total de champignons d'origine terrestre dans l'eau du lac-réservoir SMBA a été estimé à $10250 \mathrm{UFC} /$ litre. Ce résultat est la moyenne des dénombrements réalisés sur les échantillons d'eau provenant des quatre sites d'échantillonnage.

De la comparaison des résultats du dénombrement, il ressort que l'eau superficielle du rivage est plus chargée en propagules fongiques (20 $750 \mathrm{UFC} /$ litre) que celle du fond à $0,5 \mathrm{~m}$ de profondeur (10 $800 \mathrm{UFC} /$ litre). Alors qu'au niveau du point représentatif, l'eau à 
un mètre du fond (5 $625 \mathrm{UFC} /$ litre) est plus riche en champignons que celle de la thermocline (3 $825 \mathrm{UFC} /$ litre). Ces résultats montrent aussi que l'eau du rivage renferme plus de champignons que celle du point représentatif. La richesse du rivage en éléments fongiques s'explique par la proximité de ce dernier au milieu extra-aquatique qui est le pourvoyeur du milieu aquatique en organismes terrestres.

\section{3-2. Inventaire des micromycètes}

Les travaux de détermination réalisés sur les champignons isolés de l'eau ont révélé la présence de cinquante espèces fongiques. Les souches identifiées sont des Champignons Imparfaits et des Ascomycètes; elles appartiennent principalement aux genres Penicillium, Fusarium, Aspergillus et Trichoderma. Les différentes espèces identifiées et leurs lieux d'isolements sont consignés dans le Tableau $I$.

Tableau 1 : Liste des espèces fongiques isolées de l'eau du lac-réservoir SMBA.

\begin{tabular}{|c|c|c|c|c|}
\hline \multicolumn{2}{|c|}{ Espèces fongiques } & \multirow{2}{*}{$\begin{array}{l}\text { Lieux d'isolement } \\
\text { thermocline }^{(3)}\end{array}$} & \multirow{2}{*}{$\begin{array}{l}\text { Milieux d'isolement } \\
\text { eau }\end{array}$} & \multirow{2}{*}{$\begin{array}{l}\text { Champignons déjà } \\
\text { isolés du Maroc(1) ou } \\
\text { d'Algérie }^{(2)} \\
\text { (1) ef (2) }\end{array}$} \\
\hline & roseum(Oud.) W. Gams & & & \\
\hline & terricola (Miller \& al.) W. Gams & thermocline & eav & \\
\hline \multirow{6}{*}{ Aspergillus } & flavus Link & surface rivage ${ }^{(4)}$ & eau & (1) et (2) \\
\hline & niger van Tieghem & $\begin{array}{l}\text { fond rivage }{ }^{(4)} \text {, thermocline, } \\
\text { fond } \mathrm{PR}^{(3}\end{array}$ & $\begin{array}{l}\text { eau, feville du fond } \\
\text { rivage }\end{array}$ & (1) ef (2) \\
\hline & ochraceus Wilhelm & surface rivage & écume & (1) ef (2) \\
\hline & petrakiiVoros & surface rivage & écume & (1) et (2) \\
\hline & terreus Thom & surface rivage & eau & (1) ef (2) \\
\hline & versicolor(Vuill.) Tiraboschi & surface rivage & eau & (1) \\
\hline Bipolaris & australiensis(Ellis) Tsuda \& Ueyama & fond rivage & feville & (1) et (2) \\
\hline Cladosporium & sphaerospermum Penz. & rivage : surface et fond & $\begin{array}{l}\text { eau fond rivage, } \\
\text { écume }\end{array}$ & (1) \\
\hline Coniothyrium & cerealis E. Mull. & surface rivage & eau & \\
\hline Curvularia & pallescens Boedijn & surface rivage & écume & \\
\hline Cylindrocarpon & didymum(Hartig) Wollenw. & rivage : surface et fond & branches, écume & (ylindrocarpon sp.(1) \\
\hline Cytospora & $s p$. Ehrenb. & surface rivage & eau & (1) ef (2) \\
\hline Drechslera & $\begin{array}{l}\text { dematioidea (Bubak \& } \quad \text { Wroblewski) } \\
\text { Subramanian \& Jain }\end{array}$ & fond rivage & feuille & \\
\hline Engyodontivm & album(Limber) de Hoog & surface rivage & eau & \\
\hline Epicoccum & purpurascens Ehrenb. ex Schlecht & rivage: surface et fond & eau fond, écume & \\
\hline
\end{tabular}




\begin{tabular}{|c|c|c|c|c|}
\hline \multicolumn{2}{|c|}{ Espèces fongiques } & \multirow{2}{*}{$\begin{array}{l}\text { Lieux d'isolement } \\
\text { fond rivage }\end{array}$} & \multirow{2}{*}{$\begin{array}{l}\text { Milieux d'isolement } \\
\begin{array}{l}\text { sédiment organique } \\
\text { branches }\end{array}\end{array}$} & \multirow[t]{2}{*}{$\begin{array}{l}\text { Champignons déjà } \\
\text { isolés du Maroc(l) ou } \\
\text { d'Algérie(2) }^{(2)}\end{array}$} \\
\hline \multirow{7}{*}{ Fusarium } & fusarioides(Frag. \& (if.) Booth & & & \\
\hline & equiseti(Corda.) Sacc. & fond rivage & feuille & \\
\hline & lateritium Nees & fond rivage & feuille & \\
\hline & oxysporum Schlecht & fond rivage & feuille & (1) ef t(2) $^{(2)}$ \\
\hline & sambucinum Fuckel & fond rivage & sédiment organique & \\
\hline & solani(Mart.) Sacc. & fond rivage & branches & ${ }^{(1)} \mathrm{et}^{(2)}$ \\
\hline & ventricosum Appel \& Wollenweber & fond rivage & feuille & \\
\hline Paecilomyces & lilacinus(Thom) Samson & surface rivage & eau & (1) \\
\hline \multirow{11}{*}{ Penicillium } & chrysogenum Thom & fond rivage & feuille & (1) \\
\hline & citreonigrum Dierckx & fond rivage & eau & \\
\hline & crustosum Thom & surface rivage & eau & (1) et ${ }^{(2)}$ \\
\hline & expansum Link & $\begin{array}{l}\text { surface rivage, thermocline, fond } \\
\text { PR }\end{array}$ & eau, écume & (2) \\
\hline & glabrum(Wehmer)Westling & thermocline, fond PR & eau & \\
\hline & janthinellum Biourge & surface rivage & eau & (1) et ${ }^{(2)}$ \\
\hline & minioluteum Dierckx & surface rivage & eau & ${ }^{(1)}$ ef $^{(2)}$ \\
\hline & oxalicum Currie \&Thom & surface rivage & eau & (1) et $^{(2)}$ \\
\hline & pinophilum Hedgcock & $\begin{array}{l}\text { surface rivage, thermocline, fond } \\
\text { PR }\end{array}$ & eau & (2) \\
\hline & simplicissimum(Oudem.) Thom & surface rivage & eau & \\
\hline & spinulosum Thom & $\begin{array}{l}\text { surface rivage, fond rivage, } \\
\text { thermocline }\end{array}$ & eau & \\
\hline \multirow{2}{*}{ Phoma } & befae Frank & surface rivage & eau & Phoma sp.(1) \\
\hline & putaminum Speg. & surface rivage & écume & (2) \\
\hline Rhodotorula & aurantiaca(Saito) Lodder & rivage : surface et fond & $\begin{array}{l}\text { eau surface rivage, } \\
\text { sédiment organique }\end{array}$ & (1) $\mathrm{et}^{(2)}$ \\
\hline \multirow{3}{*}{ Talaromyces } & flavus(Klocker) Stolk \& Samson & thermocline & eau & (1) et (2) $^{(2)}$ \\
\hline & stipitatus(Thom) C.R. Benjamin & fond PR & eau & (1) ef ${ }^{(2)}$ \\
\hline & trachyspermus(Shear) Stolk \& Samson & fond PR & eau & (1) ef ${ }^{(2)}$ \\
\hline \multirow{6}{*}{ Trichoderma } & aureoviride Rifai & surface rivage & écume & \\
\hline & hamatum(Bon.) Bain. & fond rivage & sédiment organique & (2) \\
\hline & harzianum Rifai & fond rivage & $\begin{array}{l}\text { sédiment organique ; } \\
\text { branches, feuille }\end{array}$ & (1) ef ${ }^{(2)}$ \\
\hline & koningiiOud. & surface rivage & écume & ${ }^{(1)} \mathrm{ef}^{(2)}$ \\
\hline & pseudokoningii Rifai & fond PR & eau & \\
\hline & viride Pers. ex S.F. Gray & fond rivage & sédiment organique & (1) ef (2) $^{(2)}$ \\
\hline \multirow[t]{2}{*}{ Ulocladium } & atrum Preuss & fond rivage & $\begin{array}{l}\text { sédiment organique, } \\
\text { branches, feuille }\end{array}$ & \\
\hline & chartarum(Preuss) Simmons & fond rivage & sédiment organique & (1) \\
\hline
\end{tabular}


'. Champignons isolés de l'oved Sebou au Maroc par Sage et al. [12].

2. Champignons isolés par Chafai [28] de sédiments d'oueds et d'effluents industriels de I'Est algérien.

${ }^{3}$. La thermocline et le fond PR sont localisés au niveau du point représentatif à environ 9 $m$ de profondeur et $\dot{a} I m$ du fond, respectivement.

${ }^{4}$. Les sites (surface rivage) et (fond rivage) sont situés au niveau du rivage, le premier d̀ la surface de l'eau et le second au fond à $0,5 \mathrm{~m}$ de profondeur.

5. Sédiment organique : certaines espèces ont été isolées d'une couche de matière organique, d'environ un cm d'épaisseur, déposée sur le fond du rivage.

Ce sont généralement des champignons telluriques à large répartition géographique. Ils vivent en saprophytes sur la matière organique en décomposition ou en parasites sur les végétaux ou les animaux.

De l'examen du Tableau $I$, il se dégage que l'eau de rivage est généralement chargée en propagules des genres Penicillium (10 espèces), Fusarium (7 espèces) et Aspergillus (6 espèces). Quant aux eaux profondes de la thermocline et du fond, elles sont principalement riches en Talaromyces (3 espèces) et Acremonium (2 espèces). Les champignons associés à la matière organique en décomposition dans l'eau appartiennent surtout aux genres Fusarium, Ulocladium et Trichoderma.

L'écume a la particularité de piéger les spores des champignons dérivant dans l'eau ; les cultures ensemencées à partir de cette matière ont permis d'isoler de nombreux micromycètes: Aspergillus ochraceus, A. petrakii, Cladosporium sphaerospermum, Curvularia pallenscens, Cylindrocarpon didymum, Epicoccum purpurascens, Penicillium expansum, Phoma putaminum, Trichoderma aureoviride et $T$. konongii. La majorité des conidies présentes dans les écumes provient du milieu aquatique ou de son proche environnement [22] ; certaines sont apportées par l'air et d'autres appartiennent à des Hyphomycètes terrestres comme Alternaria, Beltrania, Cladosporium, Dreschlera et Epicoccum [23].

Park [24] a classé les différents types de microorganismes présents dans l'eau douce en fonction des activités qu'ils mènent dans ce milieu; d'après cette classification, les champignons peuvent être classés en 3 groupes : les aquatiques vrais, les occasionnels et les champignons en survie. Les micromycètes isolés de la retenue SMBA peuvent être qualifiés de champignons en survie dans l'eau. Ce sont des champignons terrestres qui arrivent dans le milieu aquatique par le biais du vent ou des ruissellements, mais leurs 
activités commencent immédiatement à diminuer à cause du changement des conditions du milieu. Ils s'y retrouvent quelquefois loin de leur origine, survivant pendant des temps variables et sont ensuite redistribués avec l'eau dans la nature. Des chercheurs de notre laboratoire ont déjà isolé de l'eau de ce lac 5 champignons aquatiques vrais appartenant aux espèces Pythium diclinum [25], $P$. torulosum, $P$. catenulatum, $P$. "Group $F "[26]$ et Achlya abortispora [27], qui est une nouvelle espèce.

\section{3-3. Comparaison avec les micromycètes isolés d'autres sites}

L'examen de la liste des espèces du Tableav I, à la lumière des micromycètes recensés dans d'autres endroits du Maroc ou d'Algérie, a permis de constater une importante similitude entre les listes comparées. Ainsi Sage et al. [12] ont isolé une centaine d'espèces fongiques au cours de leur étude sur la biodiversité de la mycoflore en fonction de la pollution de l'oued Sebou. Ce dernier, situé dans la même région du Maroc que le lac-réservoir SMBA, connaît une forte pollution. De la comparaison des champignons isolés des deux sites (Tableau 1 ), il ressort que $52 \%$ des espèces isolées de la retenue SMBA sont communes à celles de l'oued Sebou.

L'analyse mycologique des sédiments d'oueds et d'effluents industriels de l'Est algérien par Chafai [28] a montré une microflore fongique assez riche formée de 96 espèces de micromycètes. Huit espèces ont été observées dans la presque totalité de leurs prélèvements ; ce sont Trichoderma harzianum, T. koningii, Fusarium solani, Aspergillus niger, A. terreus, A. fumigatus, Gliocladium virens et Mucor hiemalis. Toutes ces espèces, à l'exception des trois dernières, ont été retrouvées dans le lac-réservoir SMBA.

Plusieurs variétés de Fusarium oxysporum responsables de fusariose vasculaire de plantes ont été isolées au Maroc. Ce sont, à titre d'exemple, la variété responsable du bayoud du palmier dattier [11, 29] et la variété pathogène de la lentille [30]. Cette espèce a été aussi prélevée de la retenue enquise.

A notre connaissance, les 6 espèces suivantes sont inventoriées pour la première fois au Maroc: Curvularia pallescens, Drechslera dematioidea, Engyodontium album, Penicillium citreonigrum, Penicillium simplicissimum et Trichoderma aureoviride.

\section{Conclusion}

Cette contribution à la connaissance des micromycètes du Maroc a montré que l'eau d'un lac-réservoir situé à Rabat renferme de nombreux champignons d'origine terrestre ; ces mycètes sont particulièrement abondant dans l'eau du rivage de la retenue. 
L'inventaire des micromycètes isolés présente principalement des Champignons Imparfaits et des Ascomycètes de type saprophyte ou parasite ; ils parviennent dans le milieu aquatique ef y restent en survie pendant des temps variables.

Des travaux sont envisagés sur la capacité de certains micromycètes à se développer sur un sédiment de nature organique présent dans le lac. II s'agit de vérifier leur aptitude à décomposer la matière morte et à produire des substances indésirables, comme les toxines et les composés responsables de flaveurs de l'eau.

\section{Remerciements}

Recherches menées dans le cadre du Programme Thématique d'Appui à la Recherche Scientifique (PROTARS I) du Maroc et du Comité franco-marocain (Action Intégrée $n$ : MA/02/5I).

\section{Références}

[1-4] - G. MALENÇON «Prodrome d'une flore mycologique du Moyen-Atlası, $1^{\circ}-4^{\circ}$ Contributions, Bull. Soc. Mycol. France, (1): 68 (1952) 297-326; (2) : 70 (1954) 117-156; (3) : 71 (1955) 265-311; (4) : 73 (1958) 289-330.

[5,6] - A.L. GUYOT et G. MALENÇON «Urédinées du Marocll, I, II. Travaux de l'Institut Scientifique Chérifien, Série botanique, 11 (1957) V + 184 pp.; 28 (1963) VIII + 161 pp.

[7] - G. MALENÇON et BERTAULT R, "Flore des champignons supérieurs du Maroc", Tome I, Ed. Faculté des Sciences de Rabat, Maroc. (1970).

[8] - G. MALENÇON et R. BERTAULT, "Flore des champignons supérieurs du Maroc', Tome II, Travaux de l'Institut Scientifique Chérifien et de la Faculté des Sciences de Rabat, Maroc. (1975).

[9] - M. BESRI ॥Phases de la transmission du Fusarium oxysporumf. sp. Lycopersiciet de Verticillium dahliae par les semences de quelques variétés de tomatell, Phytopathologische Zeitschrift, 93, (1978) 148-163.

[10] - M. BESRI IIInfluence de la température du sol et de l'air sur la fusariose et la verticillose des tomates d'automne et de printemps au Marocll, Phytopathologische Zeitschrift, 98, (1980) 97-107. 
[11] - M. DJERBI, MY.H. SEDRA et M.A. EL IDRISSI ॥Caractéristiques culturales et identification du Fusarium oxysporum f. sp. albedinis, agent causal du bayoudı, Anal. inst. natn. rech. agro. (Tunisie) 1, (1985) 1-8.

[12] - L. SAGE, L. BENNASSER, R. STEIMAN and F. SEIGLE-MURANDI "Fungal microflora biodiversity as a function of pollution in oued Sebou (Morocco)II, Chemosphere, 35 (4) (1997) 751-759.

[13] - AFNOR, "Directives générales pour le dénombrement des levures et moisissures", NF ISO 7954, Ed. AFNOR, Paris. (1988).

[14] - C. B00TH, "The genus Fusarium", Ed. Commonwealth Mycological Institute, Kew, England (1971).

[15] - G.S. DE HOOG and J. GUARRO, "Atlas of Clinical Fungi", Ed._CBS. Barcelona (1995).

[16] - K.H. DOMSCH, W. GAMS and T.-H. ANDERSON, "Compendium of soil fungi", Ed. Academic Press, London (1980).

[17] - B.M. ELLIS, "Dematiaceous hyphomycetes", Ed. Commonwealth Mycological Institute, Kew, England (1971).

[18] - B.M. ELLIS, "More dematiaceous hyphomycetes", Ed._Commonwealth Mycological Institute, Kew, England (1976).

[19] - J. I. PITT, "The genus Penicillium and its teleomorphic states Eupenicillium and Talaromyces", Ed. Academic Press, London (1979).

[20] - K. B. RAPER and D.I. FENNELL, "The genus Aspergillus", Ed. Krieger, New York (1973).

[21] - M. A. RIFAI "A revision of the genus Trichoderman", Mycol. Papers, No. 116 (1969).

[22] - C.T. INGOLD, "An illustrated guide to aquatic and water-borne Hyphomycetes (Fungi Imperfecti) with notes on their biology", Ed. Sci. Publ. No. 30, Freshwater Biological Association (1975).

[23] - T.K. GOH and K.D. HYDE «Biodiversity of freshwater fungill, J. Ind. Microbiol. 17 (1996) 328-345.

[24] - D. PARK ॥On the ecology of heterotrophic microorganisms in fresh-waterll, Trans. Br. Mycol. Soc. 58 (1972) 291-299.

[25] - A. El ANDROUSSE, A. El AISSAMI, M. RAHOUTI, H. LAHLOU, A. BOULOUD and F. SEIGLE-MURANDI "First report of Pythium diclinum in Moroccoll, EPPO Bulletin 35 (2005) 261-264. 
[26] - A. EL ANDROUSSE, A. El AISSAMI, M. RAHOUtI, H. LAHLOU, S. BENABDELLAH and F. SEIGLE-MURANDI "First record of three species of Pythium from Moroccan watersi, Acta Bot. Malacitana 32 (2007) 1-6.

[27] - A. EL ANDROUSSE, A. EL AISSAMI and B. PAUL "Achlya abortispora, a new Oomycete isolated from water samples taken from a water reservoir in Moroccoll, Curr. Microbiol. 53 (2006) 60-67.

[28] - D. CHAFAl (1996) «Micromycètes des sédiments d'oueds et d'effluents industriels de l'Est algérienil, Th. Doctorat, Univ. Joseph Fourier, Grenoble, France, 145 p.

[29] - R.A. BRAC DE LA PERRIERE et A. BENKHALIFA IIProgression de la fusariose du palmier dattier en Algériell, Sécheresse 2, (1991) 119-128.

[30] - H. ESSALMANI et H. LAHLOU «Etude in vitro de l'activité antagoniste de quelques microorganismes à l'encontre de Fusarium oxysporum f.sp. Ientisll, Cryptogamie, Mycol. 23 (2002) 221-234. 